\title{
Semiclassical Analysis of the Largest Gap of Quasi-Periodic Schrödinger Operators
}

\author{
H. Krüger ${ }^{1}$ \\ Department of Mathematics, Rice University, Houston, TX 77005, USA
}

\begin{abstract}
In this note, I wish to describe the first order semiclassical approximation to the spectrum of one frequency quasi-periodic operators. In the case of a sampling function with two critical points, the spectrum exhibits two gaps in the leading order approximation. Furthermore, I will give an example of a two frequency quasi-periodic operator, which has no gaps in the leading order of the semiclassical approximation.
\end{abstract}

Key words: gaps in the spectrum, Schrödinger operators, semiclassical analysis AMS subject classification: 47B36, 47A55

\section{Introduction}

There have been recent successes in understanding the spectrum of one frequency quasi-periodic Schrödinger operators. First, Avila and Jitomirskaya [2] have completed the proof of the so called Ten Martini Problem by showing that the spectrum of the Almost Mathieu operator is a Cantor set for all non zero couplings. Second, Goldstein and Schlag [6] have shown under a large coupling condition that for almost all frequencies an analytic sampling function generates Cantor spectrum. Third, Avila, Bochi, and Damanik [1] have shown that for a generic continuous function the quasiperiodic Schrödinger operator exhibits Cantor spectrum. These proofs have in common that they are technically involved and give no quantitative results on the size of the gaps.

My goal in this note is to compute the spectrum of quasi-periodic operators using semiclassical techniques. This has the advantage of providing quantitative bounds and being relatively easy.

${ }^{1}$ E-mail: helge.krueger@ rice.edu 
Furthermore, these results on the presence of gaps have interesting consequences for the Lyapunov exponent, see [3], the end of Section 4 in [11], or [12].

A particular case of interest is the Almost-Mathieu operator

$$
H u(n)=u(n+1)+u(n-1)+2 \lambda \cos (2 \pi \alpha n) u(n)
$$

acting on $u \in \ell^{2}(\mathbb{Z})$. If one plots the spectrum in the vertical direction and the $\alpha$ dependence in the horizontal one, one obtains a picture that resembles a butterfly, see Figure 1. Here the largest gaps in the spectrum take the role of the wings of the butterfly, see for example [8], [9], [10]. In particular [9] contains an explicit description of all gaps up to all orders in the semiclassical approximation.

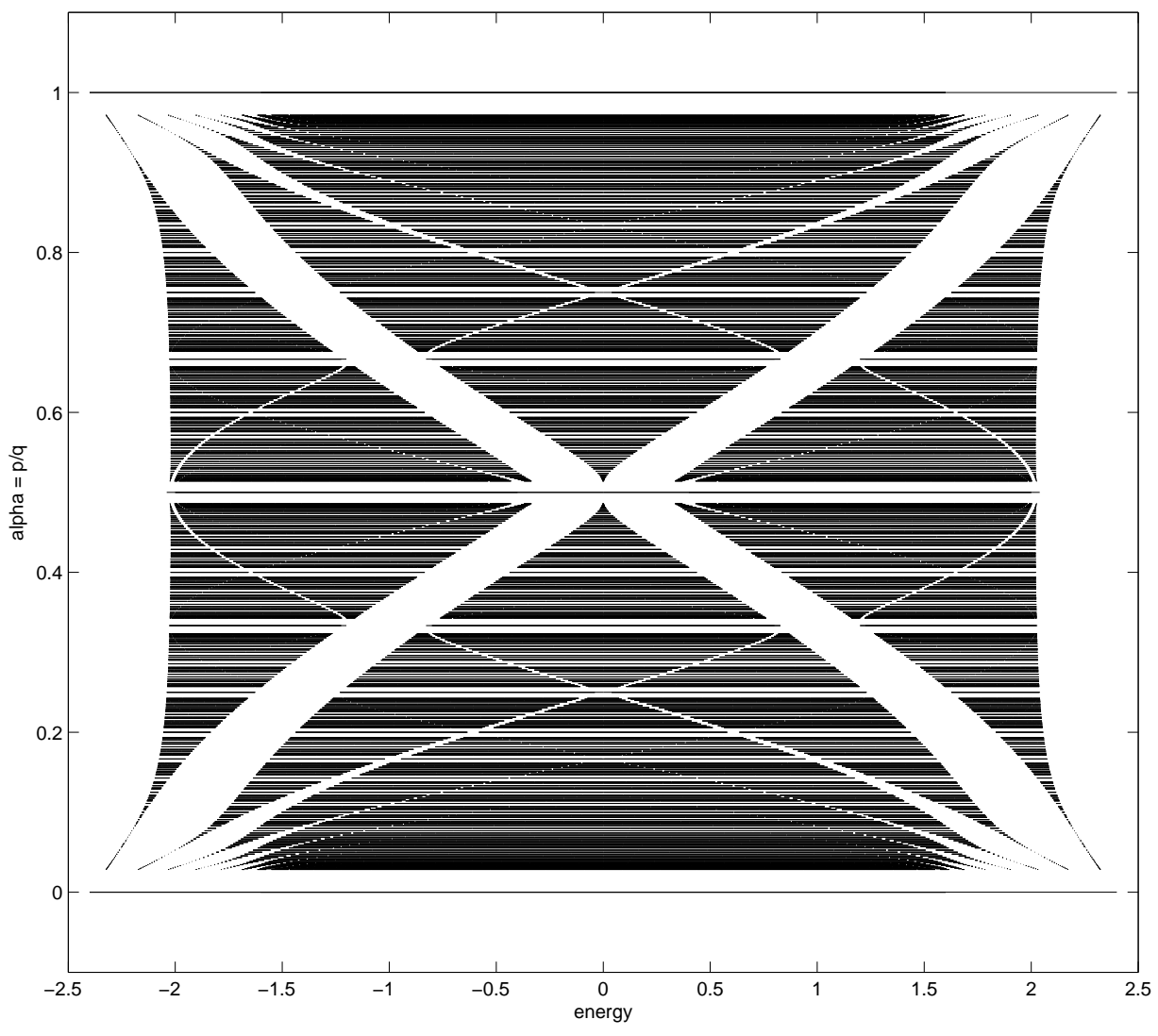

Figure 1: $\bigcup_{\theta \in[0,2 \pi]} \sigma(\Delta+2 \lambda \cos (2 \pi n \alpha+\theta))$ for $\lambda=0.2$

I will now begin to set up the problem. Given a bounded and real valued sequence $V: \mathbb{Z} \rightarrow \mathbb{R}$ 
called the potential, we introduce for $\varepsilon>0$ the family

$$
\begin{aligned}
H_{\varepsilon}: \ell^{2}(\mathbb{Z}) & \rightarrow \ell^{2}(\mathbb{Z}) \\
H_{\varepsilon} & =\varepsilon \Delta+V
\end{aligned}
$$

of discrete Schrödinger operators, where $\Delta$ is the discrete Laplacian: $\Delta u(n)=u(n+1)+u(n-1)$. Denote by $\sigma\left(H_{\varepsilon}\right)$ the spectrum of $H_{\varepsilon}$. See [13] for general background on operators of this form.

Our goal is to describe gaps, open intervals in the complement of $\mathbb{R} \backslash \sigma\left(H_{\varepsilon}\right)$, as $\varepsilon \rightarrow 0$. For simplicity, we will assume that the range of $V$ consists of a single interval. An open interval $\left(E_{-}, E_{+}\right)$is a gap of $H_{\varepsilon}$ if

$$
\left[E_{-}, E_{+}\right] \cap \sigma\left(H_{\varepsilon}\right)=\left\{E_{-}, E_{+}\right\} .
$$

The following can be shown using perturbation theory. Assume that $\left(E_{-}, E_{+}\right)$is a gap of $H_{\varepsilon_{0}}$, then one can choose continuous functions $E_{ \pm}(\varepsilon)$ such that

$$
E_{ \pm}\left(\varepsilon_{0}\right)=E_{ \pm}
$$

and $\left(E_{-}(\varepsilon), E_{+}(\varepsilon)\right)$ is gap of $H_{\varepsilon}$ in some open range of $\varepsilon$. We will be interested in gaps, where 0 belongs to the closure of this range. Since $\|\Delta\| \leq 2$, we have for $\varepsilon>0$ and any of these gaps

$$
0<E_{+}(\varepsilon)-E_{-}(\varepsilon) \leq 4 \varepsilon .
$$

We will describe the gaps for which there exists a constant $\kappa>0$ such that for $0<\varepsilon<\varepsilon_{0}$

$$
\kappa \varepsilon \leq E_{+}(\varepsilon)-E_{-}(\varepsilon) \leq 4 \varepsilon .
$$

In particular, we will be interested in the number of these gaps. For a gap, satisfying (1.5), call

$$
E_{c}=\lim _{\varepsilon \rightarrow 0} E_{+}(\varepsilon)=\lim _{\varepsilon \rightarrow 0} E_{-}(\varepsilon)
$$

its center.

Denote by $\mathbb{T}=\mathbb{R} / \mathbb{Z} \cong[0,1)$ the unit circle. Given a continuous function $f: \mathbb{T} \rightarrow \mathbb{R}$ and a minimal rotation $R_{\alpha}$, that is for $\alpha$ an irrational number

$$
\begin{aligned}
R_{\alpha}: \mathbb{T} & \rightarrow \mathbb{T} \\
R_{\alpha}(x) & =x+\alpha \quad(\bmod 1),
\end{aligned}
$$

we introduce the one frequency quasi-periodic potential $V$ by

$$
V(n)=f\left(R_{\alpha}^{n}(0)\right) .
$$

The choice of 0 here is arbitrary, we could as well use $V(n)=f\left(R_{\alpha}^{n}(x)\right.$ ) for any $x \in \mathbb{T}$ (see Theorem 5.21. in [13]).

It is good to keep the example of the Almost Mathieu operator in mind, when $f(x)=2 \cos (2 \pi x)$. The previous coupling constant $\lambda>0$, is now replaced by $\varepsilon=\frac{1}{\lambda}$. It is noteworthy here, that we can analyze both the small $(0<\lambda \ll 1)$ and large $(\lambda \gg 1)$ coupling regime using Aubry duality, see [3] [7].

We will show 
Theorem 1. Assume that $f$ is a twice continuously differentiable function with two critical points. Then $H_{\varepsilon}$ has two gaps satisfying (1.5).

Furthermore, if we denote by $a, b$ the two solutions of

$$
f(x+\alpha)=f(x)
$$

then we have that these two gaps have centers $f(a)$ and $f(b)$.

For example for the Almost Mathieu operator from (1.1), one obtains as centers of the gaps

$$
\pm 2 \cos (\pi \alpha) .
$$

That the assumption of 2 critical points is necessary, can be seen by

1. If $f$ had more then 2 critical points, there would be more than two solutions of equation (1.9), and thus more gaps.

2. There could be solutions of the equation

$$
f(x+\alpha)=f(x)=f(x-\alpha),
$$

which would imply the absence of gaps at energy $f(x)$, which can be seen by an adaptation of the proof of Theorem 2 .

The constant $\kappa$ in (1.5) can be bounded from below by either $s\left|f^{\prime}(a)\right|$ or $s\left|f^{\prime}(b)\right|$ for any $0<s<1$. Furthermore, the smallness condition on $\varepsilon$ depends on the size of $f^{\prime}(a)$ and $f^{\prime}(b)$ and the remainder in the Taylor expansion of $f$ around $a$ and $b$.

The methods used to prove Theorem 1 are similar to the ones used by Bourgain in [3] to describe gaps of size $\varepsilon^{2}$. I believe, one should be able to extend the methods of this paper to describe gaps of any size $\varepsilon^{k}$ as $\varepsilon \rightarrow 0$ for fixed $k \geq 1$. For the Almost-Mathieu operator, a description of the gaps of size $\varepsilon^{k}$ was obtained by Helffer, Kerdelhué, and Sjöstrand using pseudodifferential operator techniques in [9].

We will now proceed to introduce two frequency quasi-periodic potentials. Let $f: \mathbb{T}^{2} \rightarrow \mathbb{R}$ be a continuous function on the torus $\mathbb{T}^{2}$, and $R: \mathbb{T}^{2} \rightarrow \mathbb{T}^{2}$ be a minimal translations, that is for some $\alpha, \beta \in[0,1]$ satisfying $n \alpha+m \beta \neq 0(\bmod 1)$ for all integers $(m, n) \neq(0,0)$,

$$
R(x, y)=(x+\alpha, y+\beta) \quad(\bmod 1) .
$$

Introduce the potential $V$ by

$$
V(n)=f\left(R^{n}(0,0)\right)
$$

We have

Theorem 2. Let for $A>0$

$$
f(x, y)=\cos (2 \pi x)+A \cos (2 \pi y) .
$$

Then $H_{\varepsilon}$ has no gaps satisfying (1.5). 
The mechanism for to exclude gaps is more general, but one needs to be able to check that if one considers a level set

$$
S^{E}(f)=\left\{(x, y) \in \mathbb{T}^{2}: \quad f(x, y)=E\right\}
$$

then one can find a point $(x, y) \in S^{E}(f)$ such that

$$
R^{ \pm 1}(x, y) \notin S^{E}(f) .
$$

This requirement is reminiscent of the ones of Chulaevsky and Sinai in [5] under which they prove perturbatively in the rotation $R$ that the spectrum is an interval.

It should be remarked that Theorem 2 remains valid if we replace the rotation on the two dimensional torus by the skew-shift

$$
T_{\alpha}(x, y)=(x+\alpha, x+y) \quad(\bmod 1) .
$$

Showing that the spectrum of this model is actually an interval is an open problem (see Chapter 15 in [4]). In Section 2., we will relate the orbit of the skew-shift to the sequence $\frac{\alpha}{2} n^{2}(\bmod 1)$. This motivates the following theorem.

Theorem 3. Let $\alpha$ be an irrational number and $f: \mathbb{T} \rightarrow \mathbb{R}$ a continuous function, such that the level sets

$$
\{x \in \mathbb{T}: \quad f(x)=E\}
$$

consist of only finitely many points for each $E \in \mathbb{R}$. Then the Schrödinger operator with potential

$$
V(n)=f\left(\alpha n^{2}\right)
$$

has no gaps satisfying (1.5).

In the following sections, we will first prove Theoren 3 and then Theorem 2 in Section 2.. The reason for this is that verifying the non-resonance condition implying (1.5) requires some tricky manipulations in the case of Theorem 2. The proof of Theorem 1 is then given in Sections 3. and $4 .$.

\section{Proof of Theorems 2 and 3}

Given a potential $V: \mathbb{Z} \rightarrow \mathbb{R}$, we denote by $\mathcal{R}(V)$ its range

$$
\mathcal{R}(V)=\{V(n), \quad n \in \mathbb{Z}\} .
$$

For $\tau>0, E \in \mathcal{R}(V)$ is called $\tau$-non resonant, if there exists $m \in \mathbb{Z}$ such that

$$
E=V(m)
$$

and

$$
|V(m+1)-E| \geq \tau, \quad|V(m-1)-E| \geq \tau
$$

hold. Otherwise $E$ is called $\tau$-resonant. We have that 
Lemma 4. Assume that $E$ is $\tau$-non resonant. Let $\varepsilon>0$, then we have that

$$
\operatorname{dist}(E, \sigma(\varepsilon \Delta+V)) \leq \frac{\sqrt{6} \varepsilon^{2}}{\tau}
$$

Proof. Define the test vector $u \in \ell^{2}(\mathbb{Z})$ by

$$
u(n)= \begin{cases}-\frac{\varepsilon}{V(m-1)-E} & n=m-1 \\ 1 & n=m \\ -\frac{\varepsilon}{V(m+1)-E} & n=m+1 \\ 0 & \text { otherwise }\end{cases}
$$

We see that $\|u\| \geq 1$. Since $V(m)=E$, we have that

$$
((\varepsilon \Delta+V-E) u)(n)= \begin{cases}-\frac{\varepsilon^{2}}{V(m-1)-E} & n=m-2 \\ 0 & n=m-1 \\ -\varepsilon^{2}\left(\frac{1}{V(m-1)-E}+\frac{1}{V(m+1)-E}\right) & n=m \\ 0 & n=m+1 \\ -\frac{\varepsilon^{2}}{V(m+1)-E} & n=m+2 \\ 0 & \text { otherwise. }\end{cases}
$$

Since $|V(m-1)-E| \geq \tau$ and $|V(m+1)-E| \geq \tau$, we obtain that $\|(\varepsilon \Delta+V-E) u\| \leq \frac{\sqrt{6} \varepsilon^{2}}{\tau} \leq$ $\frac{\sqrt{6} \varepsilon^{2}}{\tau}\|u\|$. This implies

$$
\frac{\sqrt{6} \varepsilon^{2}}{\tau} \geq\left\|(\varepsilon \Delta+V-E)^{-1}\right\|^{-1}=\operatorname{dist}(E, \sigma(\varepsilon \Delta+V))
$$

which shows the claim.

We recall a few facts about the skew-shift. Iterating the map $T_{\alpha}$ from (1.16) gives

$$
T_{\alpha}^{n}(x, y)=\left(x+n \alpha, y+n x+\frac{n(n-1)}{2} \alpha\right) \quad(\bmod 1) .
$$

Hence, $\alpha n^{2}=p_{2}\left(T_{2 \alpha}^{n}(\alpha, 0)\right)$, where $p_{2}(x, y)=y$. Furthermore the map $T_{\alpha}$ is minimal. We are now ready to verify the non resonance condition in the case of Theorem 3.

Lemma 5. Let $f$ be as in Theorem 3 and $E_{0} \in[\min (f), \max (f)]$. There exists $\tau=\tau\left(E_{0}\right)>0$, such that for every $\varepsilon>0$, there is a $\tau$-non resonant $E$ satisfying $\left|E-E_{0}\right| \leq \varepsilon$.

Proof. Let $E_{0}=f(y)$ for some $y$, we then have that

$$
f\left(p_{2}\left(T_{2 \alpha}^{-1}\left(x, y_{0}\right)\right)=f\left(y_{0}-x+2 \alpha\right), \quad f\left(p_{2}\left(T_{2 \alpha}\left(x, y_{0}\right)\right)=f\left(y_{0}+x\right)\right.\right.
$$


for every $x \in \mathbb{T}$. Now, choose $x_{0}$ such that

$$
y_{0}-x_{0}+2 \alpha, y_{0}+x_{0} \notin f^{-1}\left(E_{0}\right),
$$

which is possible since $f^{-1}\left(E_{0}\right)$ only consists of finitely many points.

Define

$$
\tau=\frac{1}{3} \min \left(\left|E_{0}-f\left(y_{0}-x_{0}+2 \alpha\right)\right|,\left|E_{0}-f\left(y_{0}+x_{0}\right)\right|\right) .
$$

By continuity of $f$, we may find an open set $U$ such that $\left(x_{0}, y_{0}\right) \in U$ and for $(x, y) \in U$ :

$$
\left|f(y)-E_{0}\right| \leq \min (\tau, \varepsilon), \quad\left|f(y-x+2 \alpha)-E_{0}\right| \geq \tau, \quad\left|f(y+x)-E_{0}\right| \geq \tau .
$$

By minimality of $T_{\alpha}$, we may pick $m$ such that $T_{2 \alpha}^{m}(\alpha, 0) \in U$. One can now check that $E=$ $V(m)=f\left(p_{2}\left(T_{2 \alpha}^{m}(\alpha, 0)\right)\right)$ is $\tau$-non resonant.

Proof of Theorem 3. Let $E_{0} \in[\min (f), \max (f)]$ and $\tau>0$ as in the previous lemma. We may thus choose for $\varepsilon>0$ an $E$ such that

$$
\left|E-E_{0}\right| \leq \frac{\varepsilon^{2}}{\tau}
$$

Then we may compute

$$
\operatorname{dist}\left(E_{0}, \sigma(\varepsilon \Delta+V)\right) \leq d\left(E_{0}, E\right)+d(E, \sigma(\varepsilon \Delta+V)) \leq \frac{(\sqrt{6}+1) \varepsilon^{2}}{\tau} .
$$

Hence, we see that (1.5) cannot hold for any gap with center $E_{0}$, which finishes the proof.

In order to prove Theorem 2, we will need

Lemma 6. Let $f$ be as in Theorem 2 and $E_{0} \in(-1-A, 1+A)$. There exists $\tau=\tau\left(E_{0}\right)>0$, such that for every $\varepsilon>0$, there is a $\tau$-non resonant $E$ satisfying $\left|E-E_{0}\right| \leq \varepsilon$.

Proof. Introduce

$$
S=\left\{(x, y) \in\left[-\frac{1}{2}, \frac{1}{2}\right]^{2}: \quad f(x, y)=E_{0}\right\} .
$$

Since $\cos (x)=\cos (-x)$, we have that $(x, y) \in S$ implies that $(-x, y) \in S,(x,-y) \in S$, and $(-x,-y) \in S$. Because cos has two critical points, it follows that every $x$ slice contains at most two points of $S$. The same is true for the $y$ slices.

As in the last lemma, it suffices to find $\left(x_{0}, y_{0}\right) \in S$ such that

$$
\left(x_{0}+\alpha, y_{0}+\beta\right),\left(x_{0}-\alpha, y_{0}-\beta\right) \notin S .
$$

If this is the case, we introduce

$$
\tau=\frac{1}{3} \min \left(\left|E_{0}-f\left(x_{0}+\alpha, y_{0}+\beta\right)\right|,\left|E_{0}-f\left(x_{0}-\alpha, y_{0}-\beta\right)\right|\right) .
$$


To do so, we will exploit the symmetries of $S$. Let $\left(x_{0}, y_{0}\right) \in S$ and assume that (2.5) fails for $\left(x_{0}, y_{0}\right),\left(-x_{0}, y_{0}\right)$, and $\left(x_{0},-y_{0}\right)$. We will show that $\left(-x_{0},-y_{0}\right)$ satisfies (2.5).

We will assume that $\left(x_{0}+\alpha, y_{0}+\beta\right) \in S$, since the case $\left(x_{0}-\alpha, y_{0}-\beta\right) \in S$ can be handled similarly. Using the symmetry, we obtain that

$$
\left(-x_{0}-\alpha, y_{0}+\beta\right),\left(-x_{0}-\alpha,-y_{0}-\beta\right),\left(x_{0}+\alpha,-y_{0}-\beta\right) \in S .
$$

Since the $x$ and $y$ slices only contain two points of $S$, we thus obtain that $\left(-x_{0}-\alpha, y_{0}-\beta\right),\left(x_{0}-\right.$ $\left.\alpha,-y_{0}-\beta\right) \notin S$. Since $\left(-x_{0}, y_{0}\right)$ and $\left(x_{0},-y_{0}\right)$ don't satisfy (2.5), we obtain that $\left(-x_{0}+\alpha, y_{0}+\right.$ $\beta)\left(x_{0}+\alpha,-y_{0}+\beta\right) \in S$. By the symmetries of $S$, this implies that $\left(-x_{0}+\alpha,-y_{0}-\beta\right)\left(-x_{0}-\right.$ $\left.\alpha,-y_{0}+\beta\right) \in S$. Hence, we have that $\left(-x_{0}-\alpha,-y_{0}-\beta\right),\left(-x_{0}+\alpha,-y_{0}+\beta\right) \notin S$, and the claim is proven.

The proof of Theorem 2 is now completely analogous to the one of Theorem 3.

\section{Proof of Theorem 1}

To show that the gaps in Theorem 1 are open, we will need to ensure that $E=f(a), f(b)$ is never $\tau$-resonant. In fact we will need these two energies to satisfy the following stronger condition:

$E$ is $(L, \tau)$-double resonant for $V: \mathbb{Z} \rightarrow \mathbb{R}$, if there exists an $\varepsilon_{0}>0$ such that for $0<\varepsilon<\varepsilon_{0}$, there exists a set $\Lambda=\Lambda(\varepsilon) \subseteq \mathbb{Z}$ with the following properties

1. If $|V(m)-E| \leq 4 \varepsilon$, then $m \in \Lambda$.

2. For $m \in \Lambda,|V(m)-E| \leq L \varepsilon$.

3. If $m \in \Lambda$, then either $m+1 \in \Lambda$ or $m-1 \in \Lambda$.

4. If $(m, m+1) \subseteq \Lambda$, then

$$
|V(m-1)-E| \geq \tau, \quad|V(m+2)-E| \geq \tau
$$

and

$$
(V(m)-E)(V(m+1)-E) \leq 0 .
$$

We will first establish that we can satisfy any range for one frequency quasi-periodic potentials.

Lemma 7. Let $f$ and $V$ be as in Theorem 1. Then there is a constant $L>0$ and $\tau>0$ such that for $\varepsilon>0$ small enough. The two energies described in Theorem 1 are $(L, \tau)$-double resonant. Furthermore all other energies are $\tau$-non resonant for some $\tau>0$.

Proof. Since $f$ is continuous and has two critical points, its maximum $x_{+}$and minimum $x_{-}$are unique. Furthermore $f$ is strictly increasing or decreasing between these two points.

We first prove that (1.9) has exactly two solutions $a, b$. Define $g(x)=f(x+\alpha)-f(x)$. We see that $g\left(x_{+}\right)<0, g\left(x_{-}\right)>0$, and thus that (1.9) has at least two solutions $a, b$. Assume now that 
$f(a) \leq f(b)$ and for any other solution $c$ of (1.9) that $f(a) \leq f(b) \leq f(c)$ or $f(c) \leq f(a) \leq f(b)$. Consider the sets

$$
\{x: \quad f(x)<f(a)\}, \quad\{x: \quad f(x)>f(b)\} .
$$

One sees that both are intervals of length $<\alpha$. Hence, they cannot contain solutions of (1.9), and thus that there are only two solutions of (1.9).

Denote by $a, b$ the solutions of (1.9). Let $u=a$ or $u=b$ and $E=f(u)$. Introduce

$$
m=\min \left(\left|f^{\prime}(u)\right|,\left|f^{\prime}(u+\alpha)\right|\right) .
$$

Since $a$ and $b$ cannot be the maximum or minimum of $f$, we have that $m>0$. Hence, for $0<\varepsilon<$ $\frac{m}{\left\|f^{\prime \prime}\right\|_{\infty}}$ and

$$
x \notin A_{\varepsilon}=\left(u-\frac{8 \varepsilon}{m}, u+\frac{8 \varepsilon}{m}\right) \cup\left(u+\alpha-\frac{8 \varepsilon}{m}, u+\alpha+\frac{8 \varepsilon}{m}\right),
$$

we have that $|f(x)-E|>4 \varepsilon$. Define

$$
\Lambda=\left\{m: \quad m \alpha \in A_{\varepsilon}\right\} .
$$

Properties (1) and (3) are now clear from definition of $\Lambda$. Since $M=\sup _{x \in \mathbb{T}}\left|f^{\prime}(x)\right|<\infty$, we see that (2) holds with $L=\frac{8 M}{m}$. The second part of (4) follows from $f^{\prime}(u) \cdot f^{\prime}(u+\alpha)<0$.

Since $\alpha$ is irrational, we can ensure by choosing $\varepsilon$ small enough that

$$
u+2 \alpha, u-\alpha \notin A_{\varepsilon} .
$$

This implies the first part of 4 , since $A_{\varepsilon^{\prime}} \subseteq A_{\varepsilon}$ for $0 \leq \varepsilon^{\prime} \leq \varepsilon$.

Let $E=f(x)$ now be not of the above form, then we have that $f(x+\alpha) \neq E$ and $f(x-\alpha) \neq E$. This implies the $\tau$-non resonance with

$$
\tau=\frac{1}{3} \min (|f(x+\alpha)-E|,|f(x-\alpha)-E|)
$$

as in the last section. This finishes the proof.

We will show in the next section that

Proposition 8. Let $L, \tau>0$. There exists $\kappa=\kappa(L, \tau)>0$. If $E$ is $(L, \tau)$-double resonant, then there is a gap with center $E$ of size at least $\kappa \varepsilon$ for $\varepsilon>0$ small enough.

We will give the details of the proof of this theorem in the next section. Let us now proceed to Proof of Theorem 1. By the previous lemma, we can apply Proposition 8 at $E=f(a), f(b)$ and Lemma 4 otherwise. This finishes the proof. 


\section{Proof of Proposition 8}

Let $\Lambda$ be the set from the definition of double resonance. It follows from (1) that for $m \in \Lambda^{c}=\mathbb{Z} \backslash \Lambda$ we have that

$$
|V(m)-E| \geq 4 \varepsilon
$$

Introduce

$$
H_{\Lambda^{c}, \varepsilon}=\left.(\varepsilon \Delta+V-E)\right|_{\ell^{2}\left(\Lambda^{c}\right)} .
$$

We have

Lemma 9. For $0<\varepsilon<\varepsilon_{0}$, the estimate

$$
\left\|H_{\Lambda^{c}, \varepsilon}^{-1}\right\| \leq \frac{1}{2 \varepsilon}
$$

holds. Furthermore for $m$ such that $(m, m+1) \subseteq \Lambda$ and $l \in \Lambda^{c}$

$$
\begin{aligned}
\left|\left\langle\delta_{m-1}, H_{\Lambda^{c}, \varepsilon}^{-1} \delta_{l}\right\rangle\right| & \leq \frac{2}{\tau} \\
\left|\left\langle\delta_{m+2}, H_{\Lambda^{c}, \varepsilon}^{-1} \delta_{l}\right\rangle\right| & \leq \frac{2}{\tau} .
\end{aligned}
$$

hold.

Proof. In this proof all operators are thought of as acting on $\ell^{2}\left(\Lambda^{c}\right)$. By (4.1), we have that $\|(V-$ $E)^{-1} \| \leq \frac{1}{4 \varepsilon}$. Thus $\left\|\varepsilon(V-E)^{-1} \Delta\right\| \leq \frac{1}{2}$. We have

$$
H_{\Lambda^{c}, \varepsilon}=\varepsilon \Delta+V-E=(V-E)\left(\operatorname{Id}+\varepsilon(V-E)^{-1} \Delta\right),
$$

which implies the first estimate. For the second, observe that by the last equation

$$
\left\langle\delta_{m-1}, H_{\Lambda^{c}, \varepsilon}^{-1} \delta_{l}\right\rangle=\frac{1}{V(m-1)-E}\left(\sum_{k=0}^{\infty}\left\langle\delta_{m-1},\left(-\varepsilon \Delta(V-E)^{-1}\right)^{k} \delta_{l}\right\rangle\right)
$$

which implies the claim using property (4) from the definition of double resonant. The case of $m+2$ is similar.

We will now study $H_{\Lambda, \varepsilon}=\left.(\varepsilon \Delta+V-E)\right|_{\ell^{2}(\Lambda)}$. The main result is

Lemma 10. For $0<\varepsilon<\varepsilon_{0}$, we have that

$$
\left\|H_{\Lambda, \varepsilon}^{-1}\right\| \leq \frac{2+2 L}{\varepsilon} .
$$


Proof. By property (4) of double resonant, we have that

$$
H_{\Lambda, \varepsilon}=\bigoplus_{(m, m+1) \subseteq \Lambda}\left(\begin{array}{cc}
V(m)-E & \varepsilon \\
\varepsilon & V(m+1)-E
\end{array}\right) .
$$

In this formula, the $2 \times 2$ matrices are thought of as acting on $\ell^{2}(\{m, m+1\})$. An explicit computation using the second part of property (4) of double resonant yields

$$
\operatorname{det}\left(\begin{array}{cc}
V(m)-E & \varepsilon \\
\varepsilon & V(m+1)-E
\end{array}\right) \leq-\varepsilon^{2} .
$$

Now, using property (2) of double resonant, we obtain the claim.

In order to go to the whole operator, we will need the following result, which can be proven through an explicit computation.

Lemma 11 (Schur complement). Assume A is invertible. Then

$$
\left(\begin{array}{ll}
A & B \\
C & D
\end{array}\right)
$$

is invertible, if and only if

$$
S=D-C A^{-1} B
$$

is invertible. Furthermore then

$$
\left(\begin{array}{ll}
A & B \\
C & D
\end{array}\right)^{-1}=\left(\begin{array}{cc}
A^{-1}+A^{-1} B S^{-1} C A^{-1} & -A^{-1} B S^{-1} \\
-S^{-1} C A^{-1} & S^{-1}
\end{array}\right)
$$

Introduce

$$
H_{I}=\sum_{(m, m+1) \subseteq \Lambda}\left(\left\langle\delta_{m}, .\right\rangle \delta_{m-1}+\left\langle\delta_{m+1}, .\right\rangle \delta_{m+2}\right),
$$

and denote by $H_{I}^{*}$ its adjoint. We then have that

$$
\varepsilon \Delta+V-E=H_{\Lambda, \varepsilon}+H_{\Lambda^{c}, \varepsilon}+\varepsilon\left(H_{I}+H_{I}^{*}\right) .
$$

We will use the Schur complement formula with $A=H_{\Lambda^{c}, \varepsilon}, B=\varepsilon H_{I}, C=\varepsilon H_{I}^{*}$, and $D=H_{\Lambda, \varepsilon}$.

Lemma 12. For $0<\varepsilon<\varepsilon_{0}$, we have that

$$
\left\|H_{I}^{*} H_{\Lambda^{c}, \varepsilon}^{-1} H_{I}\right\| \leq \frac{8}{\tau},
$$

Proof. We compute that

$$
\begin{aligned}
H_{I}^{*} H_{\Lambda^{c}, \varepsilon}^{-1} H_{I}=\sum_{(n, n+1) \subseteq \Lambda} \sum_{(m, m+1) \subseteq \Lambda}( & \left\langle\delta_{n-1}, .\right\rangle\left\langle\delta_{m}, H_{\Lambda^{c}, \varepsilon}^{-1} \delta_{n}\right\rangle \delta_{m-1}+\left\langle\delta_{n-1}, .\right\rangle\left\langle\delta_{m+1}, H_{\Lambda^{c}, \varepsilon}^{-1} \delta_{n}\right\rangle \delta_{m+2} \\
& \left.+\left\langle\delta_{n+2}, .\right\rangle\left\langle\delta_{m}, H_{\Lambda^{c}, \varepsilon}^{-1} \delta_{n+1}\right\rangle \delta_{m-1}+\left\langle\delta_{n+2}, .\right\rangle\left\langle\delta_{m+1}, H_{\Lambda^{c}, \varepsilon}^{-1} \delta_{n+1}\right\rangle \delta_{m+2}\right) .
\end{aligned}
$$

The claim now follows by the second part of Lemma 9 . 
Introduce $S=H_{\Lambda, \varepsilon}-\varepsilon^{2} H_{I}^{*} H_{\Lambda^{c}, \varepsilon}^{-1} H_{I}$. We have that

Lemma 13. For $0<\varepsilon<\varepsilon_{1}=\min \left(\varepsilon_{0}, \frac{\tau}{16(2+2 L)}\right)$, we have that

$$
\|S\| \leq \frac{4+4 L}{\varepsilon} .
$$

Proof. Observe

$$
S=H_{\Lambda, \varepsilon}\left(\operatorname{Id}-\varepsilon^{2} H_{\Lambda, \varepsilon}^{-1} H_{I}^{*} H_{\Lambda^{c}, \varepsilon}^{-1} H_{I}\right)
$$

which implies the claim.

Combining this with the Schur complement formula, we obtain

$$
\left\|(\varepsilon \Delta+V-E)^{-1}\right\| \leq \frac{C}{\varepsilon}
$$

for some constant $C>0$. Since

$$
\left\|(\varepsilon \Delta+V-E)^{-1}\right\|=\frac{1}{\operatorname{dist}(E, \sigma(\varepsilon \Delta+V))},
$$

the proof of Proposition 8 is finished.

\section{Acknowledgements}

I am thankful to David Damanik, Michael Goldstein, and Svetlana Jitomirskaya for discussions on the subject.

\section{References}

[1] A. Avila, J. Bochi, D. Damanik. Cantor spectrum for Schrödinger operators with potentials arising from generalized skew-shifts. Duke Math. J., 146 (2009), No. 2, 253-280.

[2] A. Avila, S. Jitomirskaya. The Ten Martini Problem. Ann. of Math., 170 (2009), No. 1, 303342.

[3] J. Bourgain. Positive Lyapounov exponents for most energies. Geometric aspects of functional analysis, 37-66, Lecture Notes in Math. No. 1745, Springer, Berlin, 2000.

[4] J. Bourgain. Green's function estimates for lattice Schrödinger operators and applications. Annals of Mathematics Studies, 158. Princeton University Press, Princeton, NJ, 2005.

[5] V.A. Chulaevsky, Y. G. Sinai. Anderson localization for the 1-D discrete Schrödinger operator with two-frequency potential. Comm. Math. Phys., 125 (1989), No. 1, 91-112. 
[6] M. Goldstein, W. Schlag. On resonances and the formation of gaps in the spectrum of quasiperiodic Schrödinger equations. Ann. of Math., (to appear).

[7] A. Y. Gordon, S. Jitomirskaya, Y. Last, B. Simon. Duality and singular continuous spectrum in the almost Mathieu equation. Acta Math., 178 (1997), 169-183.

[8] J.P. Guillement, B. Helffer, P. Treton. Walk inside Hofstadter's butterfly. J. Phys. France, 50 (1989), 2019-2058.

[9] B. Helffer, P. Kerdelhué, J. Sjöstrand. Le papillon de Hofstadter revisité. Mém. Soc. Math. France (N.S.), No. 43 (1990), 87 pp.

[10] D. Hofstadter. Energy levels and wave functions of Bloch electrons in rational and irrational magnetic fields. Phys. Rev. B, 14 (1976), 2239 - 2249.

[11] H. Krüger. Probabilistic averages of Jacobi operators, Comm. Math. Phys., 295 (2010), No. 3, 853-875.

[12] H. Krüger. In preparation.

[13] G. Teschl. Jacobi Operators and Completely Integrable Nonlinear Lattices, Math. Surv. and Mon., 72, Amer. Math. Soc., Rhode Island, 2000. 